\title{
Therapeutic HIV Vaccine Trials
}

\section{Shayesta Dhalla*}

University of British Columbia, Vancouver, British Columbia, Canada

HIV vaccine research with regards to phase $3 \mathrm{HIV}$ vaccine trials has focused mainly on preventive trials. In developed countries, these include the VaxGen AIDSVAX B/B study (rgp120 vaccine), the STEP study (adenovirus-5 [Ad5] vaccine), and HVTN 505 (DNA prime with Ad5 boost). These studies have shown that the respective HIV vaccines used in these studies have been nonefficacious. However, the RV144 preventive HIV vaccine trial that took place in Thailand (ALVAC-HIV with AIDSVAX B/E boosting) showed a modest level of efficacy of the HIV vaccine of $31.2 \%$.

The phase 3 VaxGen HIV vaccine trial was conducted in the US, Canada, and the Netherlands. The phase 2B STEP study which included sites in North America and Australia, showed that there was an increased risk of HIV infection in uncircumcised men who received the Ad5 vaccine and were Ad5 seropositive, compared to those who received the placebo. The STEP study was discontinued in 2007 . The more recent HVTN 505 study that was initiated in 2009 in the US was discontinued in 2013, as the vaccine was nonefficacious and showed a non-statistically significant increased rate of infection in those who received the HIV vaccine, compared to the placebo group. Only one therapeutic phase $3 \mathrm{HIV}$ vaccine trial has been conducted, taking place in 77 US cities in mostly men, and ending in 1999. This used the HIV vaccine HIV-1 immunogen (Remune) and unrestricted antiretroviral therapy (ART), and also showed that the HIV-1 immunogen vaccine was nonefficacious. However, the study did not show an increase in clinical progression of HIV in vaccine recipients compared to placebo recipients (relative risk $[R R]=0.97,95 \% \mathrm{CI}=0.66-1.42$ ) or a difference in overall mortality between the two groups $(\mathrm{RR}=0.81,95 \% \mathrm{CI}=0.44$ 1.48).

Therefore, thus far, there have been two preventive trials that have shown an increased the rate of infection in vaccinated volunteers. Despite the promising results of the RV144 HIV vaccine trial in

\section{Thailand, this is of concern.}

The goal of therapeutic vaccination is to stimulate effective HIVspecific T cell responses, primarily CD8 T cells with CD4 T cell help, while avoiding increases in susceptibility of target cells. There are some therapeutic HIV vaccine products that for which early trails have been completed are in progress. However, a phase 3 therapeutic HIV vaccine trial could be expensive. For example, this could be due to reasons such as the costs associated with the manufacturing of the HIV vaccine, the costs associated with the implementation of an efficacy trial in the context of other therapeutic interventions, and the costs associated with measuring endpoints. Potentially, there may also be more frequent dosing in a therapeutic HIV vaccine trial. Another potential drawback of a therapeutic HIV vaccine trial is that the vaccine could increase the progression of HIV/AIDS and increase the mortality rate in HIVinfected individuals. On the other hand, the outcomes of therapeutic vaccination could be beneficial, such as counteracting resistance to ART, and counteracting drug toxicities.

Recruitment for a therapeutic trial could be challenging for a number of reasons. Secondary to the STEP study and HVTN 505 , there may therefore be a heightened sense of mistrust among potential participants. Using only specific types of populations such as circumcised men, such as in HVTN 505, could also lead to recruitment difficulties. Those who would need to be recruited in a therapeutic trial need to be HIV-infected, and may have concerns about confidentiality regarding their HIV status and participation in a clinical trial. Minority recruitment may continue to pose a challenge. The population used in the HIV vaccine therapeutic trial in the US were mostly white men (70\% in the vaccine group and $70 \%$ in the placebo group). Finding the HIV vaccine(s) to be tested is also a challenge. Despite these drawbacks, however, therapeutic HIV vaccine trials may be a viable alternative to preventive studies.
*Corresponding author: Shayesta Dhalla, University of British Columbia, Vancouver, British Columbia, Canada, E-mail: shayestadhalla@yahoo.com

Received May 23, 2013; Accepted May 27, 2013; Published May 30, 2013

Citation: Dhalla S (2013) Therapeutic HIV Vaccine Trials. J Bioanal Biomed 5 : e115. doi:10.4172/1948-593X.1000e115

Copyright: (c) 2013 Dhalla S. This is an open-access article distributed under the terms of the Creative Commons Attribution License, which permits unrestricted use, distribution, and reproduction in any medium, provided the original author and source are credited. 